\title{
Upper Limit of Radiation from Coalescence of Rotating Hayward Black Holes
}

\author{
Bogeun Gwak ${ }^{1, \star}$ \\ ${ }^{1}$ Department of Physics and Astronomy, Sejong University, Seoul 05006, Republic of Korea
}

\begin{abstract}
We review the upper limit of the gravitational radiation thermally allowed under the collision of two rotating Hayward black holes. The upper limit is dependent on the gravitational spin interaction between black holes and is useful to determine one of the parameters for consistency with Laser Interferometer Gravitational-Wave Observatory (LIGO) data.
\end{abstract}

\section{Introduction}

Colliding black holes have been an expected source of gravitational waves. The first gravitational wave, GW150914, was successfully detected at the Laser Interferometer Gravitational-Wave Observatory (LIGO)[1]. Since then, LIGO announced two more detections of gravitational waves[2, 3]. In this proceeding, we will review the upper limit of the gravitational radiation thermally allowed under the collision of two Hayward black holes[4]. Black hole thermodynamics are studied using the laws of black-hole mechanics, which are analogous to the laws of thermodynamics. They suggest that information about a black hole can be retrieved using Hawking radiation and Bekenstein-Hawking entropy. [5-8]. Particularly, the irreducibility of the entropy is important in the Penrose process[9, 10] as well as the weak cosmic censorship conjecture[11-20]. Since the collision of black holes is a natural process, we can expect an increase in the entropy of the system based on the second law of thermodynamics[21]. Fundamentally, the upper limit of the radiation depends on the interaction between black holes[22-24] and the instability of the resultant black hole formed by the collision[2528]. The Hayward black hole is a regular black hole[29], given a non-zero energy-momentum tensor in Einstein's equations. Based the weak cosmic censorship conjecture, the singularity should be hidden by the event horizon of the black hole, but the Hayward black hole has no singularity within the horizon. Using the Newman-Janis transformation[30], the rotating Hayward black hole is obtained. The metric of the Hayward black hole is also found in the gravity theory coupled with non-linear electrodynamics[31].

We review the upper limit of the gravitational radiation under the collision of two rotating Hayward black holes and the possible parameter range using LIGO data[4].

\footnotetext{
^e-mail: rasenis@ sejong.ac.kr
} 


\section{Hayward Black Hole}

We consider the coalescence of two rotating Hayward black holes. [29, 30]. Given a Hayward black hole of mass $M$ and angular momentum $J=M a$, the metric is in Boyer-Lindquist coordinates

$$
d s^{2}=-\left(1-\frac{2 m r}{\Sigma}\right) d t^{2}-\frac{4 a m r \sin ^{2} \theta}{\Sigma} d t d \phi+\frac{\Sigma}{\Delta} d r^{2}+\Sigma d \theta^{2}+\left(r^{2}+a^{2}+\frac{2 a^{2} m r \sin ^{2} \theta}{\Sigma}\right) \sin ^{2} \theta d \phi^{2},
$$

where

$$
\Delta=r^{2}-2 m r+a^{2}, \quad \Sigma=r^{2}+a^{2} \cos ^{2} \theta, \quad m=M \frac{r^{3+\rho}}{r^{3+\rho}+g^{3} \Sigma^{\rho / 2}} .
$$

The mass function $m$ has two free parameters, $\rho$ and $g$, and depends on the coordinate $\theta$ based on the function $\Sigma$. The parameter $\rho$ mainly affects the shape of the event horizon of the black hole[4]. We will use the Bekenstein-Hawking entropy to find the upper limit of the radiation. The entropy is proportional to the area of the horizon $A_{H}$, which is obtained using a numerical approach. Thus,

$$
S_{\mathrm{BH}}=\frac{1}{4} A_{H}, \quad A_{\mathrm{H}}=2 \pi \int_{0}^{\pi}\left(r_{\mathrm{h}}^{2}(\theta)+a^{2}\right) \sin \theta d \theta,
$$

which are slightly different values from that of a Kerr black hole due to the effect of additional parameters.

\section{Coalescence of Rotating Hayward Black Holes}

We will review the general process for the coalescence of black holes to obtain the upper limit of the gravitational radiation[21,22]. The upper limit will be obtained using the increased entropy of the system. This results in an inequality between the initial mass of the system and the mass of the resultant black hole. Thus, we can derive the upper limit of the radiation from the minimum mass of the resultant black hole.

\subsection{Upper Limit of Radiation}

We begin by assuming that the two Hayward black holes are far enough away from each other to eliminate the effects of interactions[4, 23-25]. Then, these black holes slowly come together and merge to form a single, resultant black hole. This is a natural process; hence, the entropy of the system will increase. We denote subscripts 1 and 2 for the initial black holes and 3 for the resultant black hole. The increase in the Bekenstein-Hawking entropy is given by the inequality

$$
A_{\mathrm{H}}\left(M_{1}, J_{1}\right)+A_{\mathrm{H}}\left(M_{2}, J_{2}\right) \leq A_{\mathrm{H}}\left(M_{3}, J_{3}\right) .
$$

where $M_{1}, M_{2}$ and $J_{1}, J_{2}$ are, respectively, the masses and angular momenta of the initial black holes. $M_{3}$ is the mass of the resultant black hole. The final angular momentum $J_{3}$ is assumed to be the total angular momentum of the system due to conservation of angular momentum; therefore,

$$
J_{3}=\sqrt{J_{1}^{2}+J_{2}^{2}+2 J_{1} J_{2} \cos \psi},
$$

where $\psi$ is the angle between the angular momentum vectors of the initial black holes. In Eq. (4), there is only one variable: $M_{3}$; therefore, we can find $M_{\min }$, the minimum value of $M_{3}$, by solving 
the inequality. The mass difference between the initial and final black holes is presumably due to gravitational radiation, which has an energy of $M_{\text {rad }}$, such that

$$
M_{\mathrm{rad}}=M_{1}+M_{2}-M_{3} .
$$

We can now estimate the minimum mass of the resultant black hole and obtain the upper limit of the radiation, $M_{\mathrm{r}}$.

$$
M_{\mathrm{r}}=M_{1}+M_{2}-M_{\min },
$$

Therefore, the actual radiation will not exceed this value for identical initial conditions.

\subsection{Parameter Range from LIGO Observations}

The rotating Hayward black hole includes several parameters. In particular, $g$ has the greatest effect on the black hole solution and upper bound of the radiation. A possible range for $g$ can be estimated using the following LIGO data: GW150914, GW151226, and GW170104[1-3]. To do so, we fix the following parameters: $\rho=0.9$ and $a=0$, since they are not as significant as $g$. The actual observations for $a$ have an error; therefore, setting $a=0$ will not affect our results for $g$. In Fig. 1, we estimate the range of $g$ using the extremal spin parameter, minimum mass, and the upper limit of the radiation. The final spin parameters of black holes are estimated; hence, the spin parameters

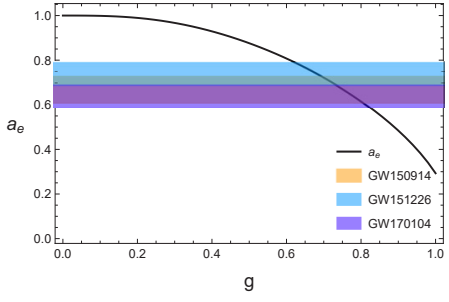

(a) The extremal spin parameter under $\rho=$ 0.9 and $M=1$.

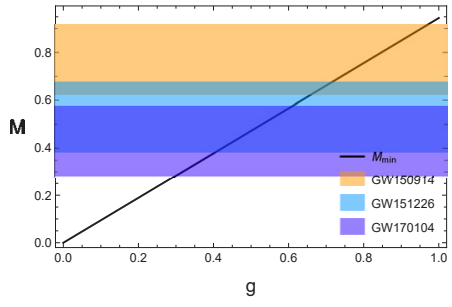

(b) The minimum mass under $\rho=0.9, M=$ 1 , and $a=0$.

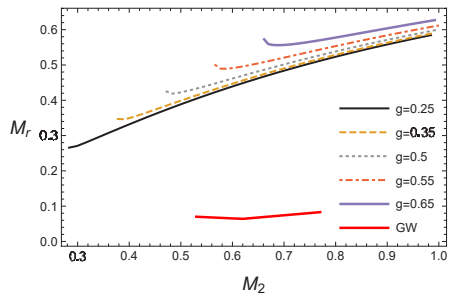

(c) The upper bounds on the radiation for $\rho=0.9, M_{1}=1, a_{1}=0$, and $a_{2}=0$, and the slope between LIGO data.

Figure 1. The upper bounds thermally allowed and detections of the LIGO .

should be within the extremal spin parameter to exist stably. In Fig. 1 (a), we show the dimensionless extremal spin parameter for a given $g$, and the straight area represents the specific observation for $a_{3}$. Accordingly, the straight area should be below the solid line to exist in our Universe. The upper bound of $g$ is approximately 0.75 . The mass ratio of the second initial black hole is greater than the extremal mass, if we set the mass of the first initial black hole to unity. In Fig. 1 (b), the minimum mass of the black hole is given in terms of each value of $g$. The upper bound of $g$ is approximately 0.99 . This value is too large to extract any meaning from it. . Using the upper limit of the radiation, as shown in Fig. 1 (c), we can see that the lines for the upper limit should span the entirety of the red line, which represents the LIGO data. Hence, the upper value of $g$ is approximately 0.5 and the possible values for $g$ are within 0 and 0.5 .

\section{Summary}

We have reviewed the upper limit of the gravitational radiation thermally allowed under the collision of two rotating Hayward black holes. We suppose an initial state in which two black holes begin 
far enough from each other, where the effects of interaction can be ignored. Then, the two black holes slowly approach one another and, finally, merge to form a resultant black hole. The entropy of the system increases, as is required by the second law of thermodynamics. Using the second law, we determine the possible minimum mass of the resultant black hole. Knowing that the total mass of the initial state is greater than that of final black hole and that mass should be conserved in spacetime, the difference in mass is presumably released as a form of gravitational radiation. Using the minimum possible mass, the upper limit of the radiation can be obtained for a given initial state. The most important parameter for the upper limit of the radiation of a rotating Hayward black hole is $g$. The upper limit can be useful to determine the possible range of $g$ using LIGO data. By using an extremal spin parameter, the minimum mass, and the upper limit of the radiation, the possible values of $g$ are within 0 and 0.5 .

\section{Acknowledgments}

This work was supported by Basic Science Research Program through the National Research Foundation of Korea (NRF) funded by the Ministry of Science, ICT \& Future Planning (NRF2015R1C1A1A02037523).

\section{References}

[1] B. P. Abbott et al. [LIGO Scientific and Virgo Collaborations], Phys. Rev. Lett. 116, no. 6, 061102 (2016).

[2] B. P. Abbott et al. [LIGO Scientific and Virgo Collaborations], Phys. Rev. Lett. 116, no. 24, 241103 (2016).

[3] B. P. Abbott et al. [LIGO Scientific and VIRGO Collaborations], Phys. Rev. Lett. 118, no. 22, 221101 (2017).

[4] B. Gwak, Eur. Phys. J. C 77, no. 7, 482 (2017).

[5] S. W. Hawking, Commun. Math. Phys. 43, 199 (1975).

[6] S. W. Hawking, Phys. Rev. D 13, 191 (1976).

[7] J. D. Bekenstein, Phys. Rev. D 7, 2333 (1973).

[8] J. D. Bekenstein, Phys. Rev. D 9, 3292 (1974).

[9] J. M. Bardeen, Nature 226, 64 (1970).

[10] R. Penrose and R. M. Floyd, Nature 229, 177 (1971).

[11] D. Christodoulou, Phys. Rev. Lett. 25, 1596 (1970).

[12] D. Christodoulou and R. Ruffini, Phys. Rev. D 4, 3552 (1971).

[13] L. Smarr, Phys. Rev. Lett. 30, 71 (1973).

[14] B. Gwak, Phys. Rev. D 95, no. 12, 124050 (2017).

[15] B. Gwak and B. H. Lee, Phys. Lett. B 755, 324 (2016).

[16] B. Gwak and B. H. Lee, JCAP 1602, 015 (2016).

[17] B. Gwak and B. H. Lee, Class. Quant. Grav. 29, 175011 (2012).

[18] B. Gwak and B. H. Lee, Phys. Rev. D 84, 084049 (2011).

[19] B. Gwak, arXiv:1707.09128 [gr-qc].

[20] B. Gwak, arXiv:1709.08665 [gr-qc].

[21] S. W. Hawking, Phys. Rev. Lett. 26, 1344 (1971).

[22] R. M. Wald, Phys. Rev. D 6, 406 (1972).

[23] B. Gwak and D. Ro, Eur. Phys. J. C 77, no. 8, 554 (2017).

[24] B. Gwak and D. Ro, arXiv:1610.04847 [gr-qc]. 
[25] B. Gwak and B. H. Lee, JHEP 1607, 079 (2016).

[26] B. Gwak, B. H. Lee and D. Ro, Phys. Lett. B 761, 437 (2016).

[27] W. K. Ahn, B. Gwak, B. H. Lee and W. Lee, Eur. Phys. J. C 75, no. 8, 372 (2015).

[28] B. Gwak and B. H. Lee, Phys. Rev. D 91, no. 6, 064020 (2015).

[29] S. A. Hayward, Phys. Rev. Lett. 96, 031103 (2006).

[30] C. Bambi and L. Modesto, Phys. Lett. B 721, 329 (2013).

[31] Z. Y. Fan and X. Wang, Phys. Rev. D 94, no. 12, 124027 (2016). 